\title{
Rapid Prototyping of an Adaptive Light-source for Mobile Manipulators with EasyKit and EasyLab.
}

\author{
Martin Wojtczyk, Simon Barner, Michael Geisinger and Alois Knoll \\ Technische Universität München, \\ Informatics VI: Robotics and Embedded Systems, \\ Boltzmannstr. 3, D-85748 Garching b. München, Germany
}

\begin{abstract}
While still not common in day-to-day business, mobile robot platforms form a growing market in robotics. Mobile platforms equipped with a manipulator for increased flexibility have been used successfully in biotech laboratories for sample management as shown on the well-known ESACT meetings. ${ }^{1}$ Navigation and object recognition is carried out by the utilization of a mounted machine vision camera. To cope with the different illumination conditions in a large laboratory, development of an adaptive light source was indispensable. We present our approach of rapid developing a computer controlled, adaptive LED light within one single business day, by utilizing the hardware toolbox EasyKit and our appropriate software counterpart EasyLab.
\end{abstract}

Keywords: Adaptive Light-source, Service Robotics, Rapid Hardware Prototyping, Zero Code Development.

\section{INTRODUCTION}

Development of mechatronic systems is a time consuming process and requires efforts in mechanical, electrical and software engineering which typically are put into effect sequentially in the mentioned order. At the chair for Robotics and Embedded Systems at the Technische Universität München we develop robot and robot part prototypes and the necessary tools to comply with the short timeline requirements. To parallelize the different development steps, we utilize the Rapid Prototyping Toolset EasyKit which consists of a collection of hardware and software modules and our model based, visual programming tool EasyLab.

One of the real world projects, where EasyKit was applied to recently, is an autonomous mobile robot platform in a Life Science Lab of a global pharmaceutical company which needed to be equipped with an adaptive lightsource to improve illumination conditions for its image processing. Figure 1 shows the lab robot and a close-up of its tool. The robot is able to carry out a complete sample management process in a pilot plant, as shown previously at the ESACT meeting 2005. Since the robot moves to different stations and analysis devices in a large laboratory, illumination conditions vary according to the time of the day, and according to the distance to the windows and the overhead lights. While the image processing performed perfectly in well illuminated areas, we wanted the robot to be ready also for challenging environments. Several more difficult scenarios are apparent and were encountered: the overhead lights in the lab might just accidentally be switched off by a human, a power outage may occur and in worst case there would be no daylight late at night to illuminate the environment or due to the absence of windows.

This was the driving force to equip the robot with an adaptive, computer-controlled light source to locally illuminate the area of interest according to ambient light conditions. We wanted the light source to be computercontrolled, since different materials have different reflection properties and may cause problematic highlights if the light is just switched on to maximum power.

Further author information: (Send correspondence to Martin Wojtczyk) Martin Wojtczyk: E-mail: wojtczyk@in.tum.de, Telephone: +49-89-289-18143

Simon Barner: E-mail: barner@in.tum.de, Telephone: +49-89-289-18111

Michael Geisinger: E-mail: geisinge@in.tum.de, Telephone: +49-89-289-18111

Alois Knoll: E-mail: knoll@in.tum.de, Telephone: +49-89-289-18104 

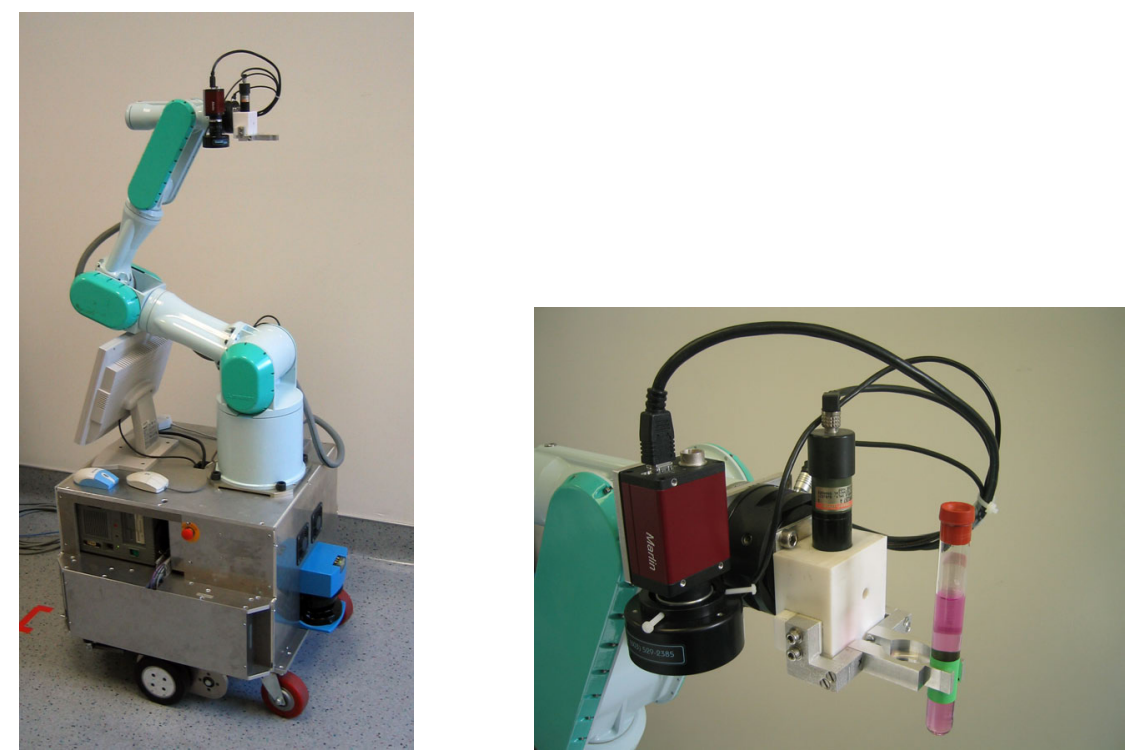

Figure 1. The mobile robot platform for automation of sample management processes. The close-up picture on the right shows the robot arm's tool with the red camera pointing down for object detection and the two fingered gripper for grasping vials of different sizes.

\section{MOTIVATION}

Before we started development, we carried out a literature and parts research, not to reinvent the wheel. The requirements for our light-source were the following:

- Computer-controlled brightness

- Low energy consumption

- Easily attachable to the robot's tool

- As cheap as possible

Since the robot is battery driven, we wanted the light source not to consume too much energy. Furthermore it should fit around the camera to illuminate the region which the robot is looking at and its brightness should be controllable on demand by the robot's on-board computer. Finally, also the price is an important factor.

During parts research we found an appropriate LED array, circular in shape, with 24 or alternatively 48 white LEDs, which perfectly fits around machine vision lenses and whose energy consumption is small due to the utilization of LEDs. Siemens/NERLITE provides these LED arrays for machine vision applications as offthe-shelf components. ${ }^{2-4}$ The power requirements of the selected ring lights are $12 \mathrm{~V} / 160 \mathrm{~mA}$ for the 24 LED R-60-1 "V2" and 24 V/160 mA for the 48 LED R-70-2 "V2". The lights are available with different delivery rings, out of which we chose the diffuse ones for a homogenous illumination.

The brightness of LED arrays can be controlled by adjusting the current, which apparently also changes the wavelength of the emitted light or - which is the commonly preferred method - by powering the LEDs with a pulse width modulated signal, where a low frequency will result in low brightness while a high frequency results in a brighter light. During parts research we came across several components, however after intensive investigation we only found one controller which can be directly connected to a computer via RS232: the Gardasoft Vision PP610 - LED Lighting Controller with RS232 Control. ${ }^{5,6}$ However at a price of 866 EUR, we double-checked, if it was possible to create an appropriate, computer interfaceable controller by ourselves utilizing our Rapid Hardware Prototyping Toolset EasyKit, which resulted in this proposal. ${ }^{7}$ 


\section{BACKGROUND}

This section shortly describes the autonomous lab robot and EasyKit, consisting of a modular Hardware Toolbox and the visual software development Toolbox EasyLab for a better understanding.

\subsection{Robot}

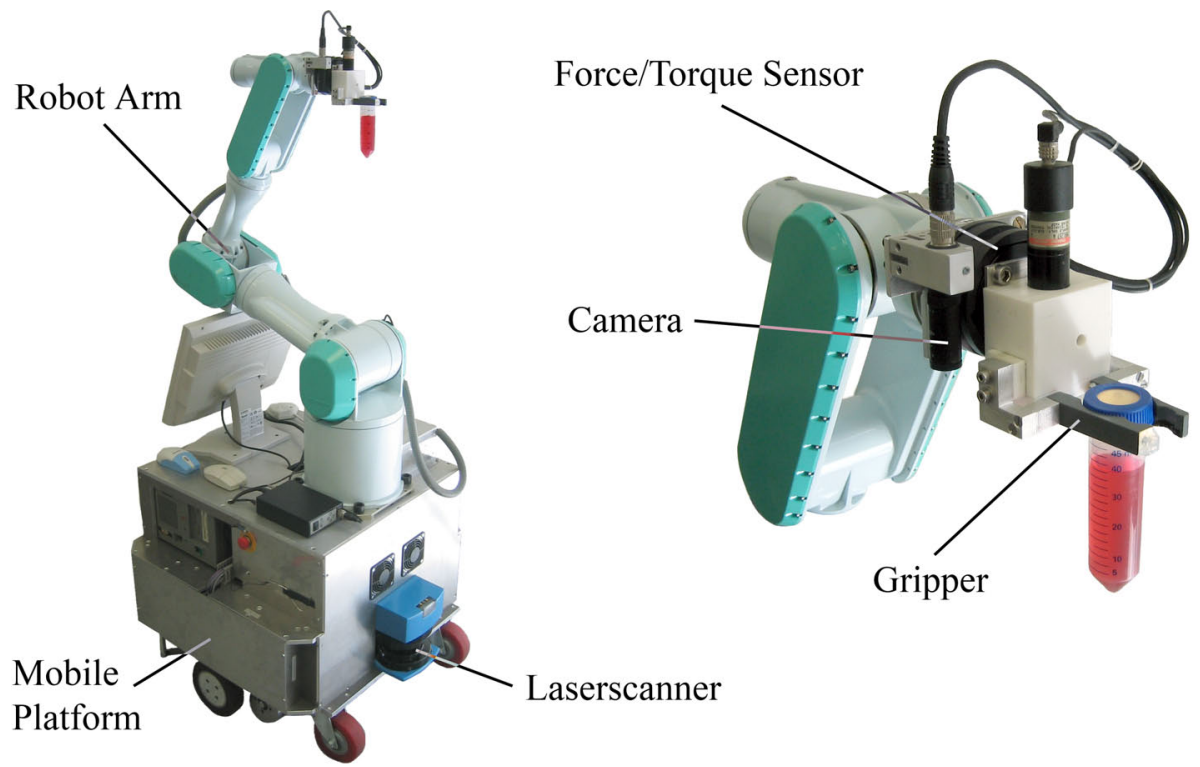

Figure 2. The mobile robot platform with its sensors and a close-up of its tool.

A mobile robot system, previously developed at the University of Bielefeld, Germany is capable of automating the complete sample management in a biotechnological laboratory (see Figures 1 and 2). The use of this autonomous robot, operating several distinct analysis and sampling devices, resulted in a fully automated sample management system, available day and night and tested successfully in pilot scale.

The robot consists of a battery driven, wheeled platform and a mounted industrial robot arm with a twofinger gripper attached to it. The proper interaction with the analysis and sample devices in the biotech lab is given by the use of a color camera for object recognition, a force/torque sensor to prevent damages and laser range finders at the front and the backside of the robot for localization. The on-board computer is responsible for localizing and navigating the platform with respect to the sensor data from the laser range finders. Furthermore the computer controls the mounted robot arm's movements. Precise device interactions with an accuracy of less than $1 \mathrm{~mm}$ are performed by pattern and object detection, utilizing the camera and dedicated computer vision algorithms in combination with realtime sensor feedback of a force-/torque sensor in between the robot's tool and its arm..$^{8-11}$

\subsection{Hardware Toolbox}

Mechatronic applications often utilize many different Integrated Circuits (IC) which need to be arranged and assembled on a circuit board and connected to data interfaces. For rapid prototyping EasyKit includes a modular toolbox based on Match-X with different circuit blocks, which can be easily put together. Figure 3 exemplary shows some components out of the construction kit and the connection through the standardized electrical data bus.

The available modules cover a variety of requirements and provide a CPU block with a Microchip PIC 18F2520 Microcontroller ${ }^{12}$ as well as a voltage regulator, a serial interface component and sensor connector components among others. The Match-X standard is published by the VDMA and also covers the development transition from Match-X blocks towards small batch and series production. ${ }^{13,14}$ 

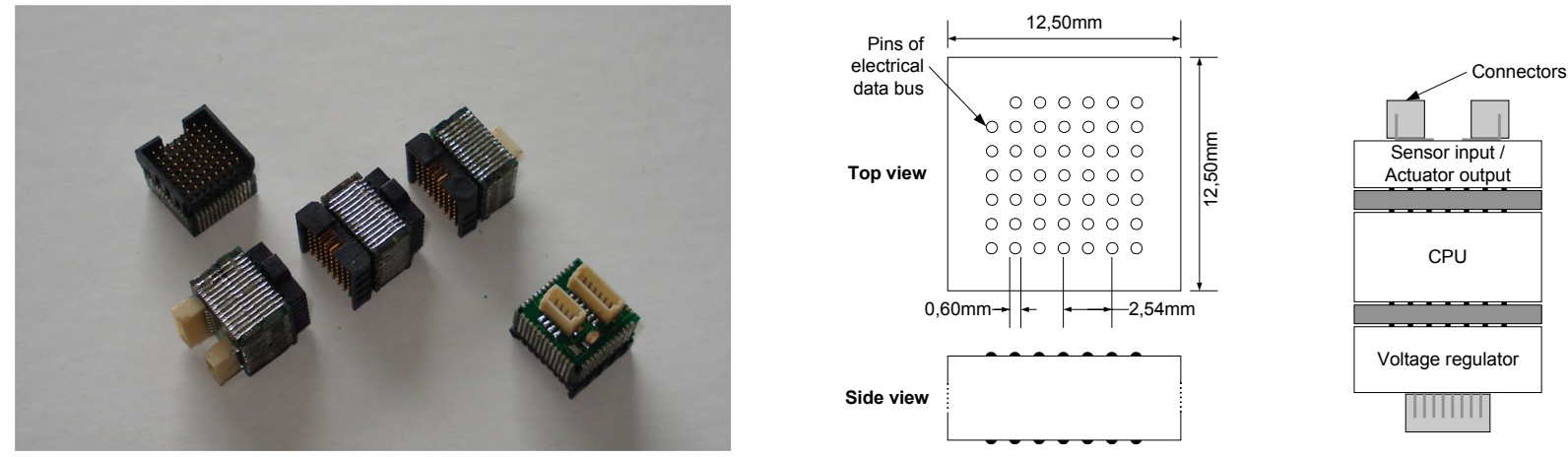

Figure 3. Some exemplary modules out of the Match-X construction kit are depicted on the left, while the center image shows the data bus layout. The right image shows an elementary assembly of three blocks with a CPU, a voltage regulator and sensor connectors.

\subsection{Software Toolbox}

EasyLab is a modular and model based Integrated Development Environment (IDE) for creating software applications focussing on but not limited to embedded systems. It provides two kinds of graphical software models: Structured Flow Charts (SFC) and Synchronous Data Flows (SDF). ${ }^{15-19}$ SFCs describe the different possible states of a system and the state transitions. The states themselves are references to subprograms implemented in any arbitrary language, while the transitions can be alternative branches conditioned by boolean expressions and parallel branches. A state itself can be another SFC or for example a SDF, which is a directed multigraph, consisting of actors connected by edges, representing the data flow between actors.

The functionality of hardware modules such as described in 3.2 is represented by analogous software counterparts with the appropriate input and output connectors for SDFs. In addition to that, the IDE also provides building blocks for generic data types and methods to define constants, variables, arrays and images for example as well as common operations like mathematical functions or image processing filters, just to mention a few of them.

Figure 4 shows a sample application implemented in EasyLab. The left picture shows an SFC with a sequence of different states, which in this case, represent several brightness values of the light source. The transition is performed when the boolean expression becomes true, hence when the utilized counter expires. The SDF in the right picture shows a simple assignment subroutine, where a constant value is assigned to the counter. As a result, the ring light will sequentially change it's brightness after a certain waiting period, which is determined by the counter, passed.

After defining the application logic in EasyLab, the user can invoke the integrated code generator to create customized machine code for the targeted microcontroller, which in turn is usually limited in its resources. Compared to the method to write microcontroller applications from scratch - which still is a common and widely used approach - the utilization of EasyLab can reduce development time dramatically.

\section{IMPLEMENTATION}

Our goal was to develop an adaptive, computer-controlled light-source for a mobile manipulator to improve illumination conditions for the mounted camera and the computer vision system. As Hardware components we decided to use the Siemens/NERLITE ring illuminators mentioned in 1 and a controller stack built from Match-X components. The solution for this task was as follows:

Initially the robot takes an image with its onboard camera. Afterwards the brightness of the acquired image is determined by calculating a brightness histogram and its mean in a range from 0 to 255. A controller in the host application gradually increases or decreases the brightness of the ringlight by sending brightness values to 

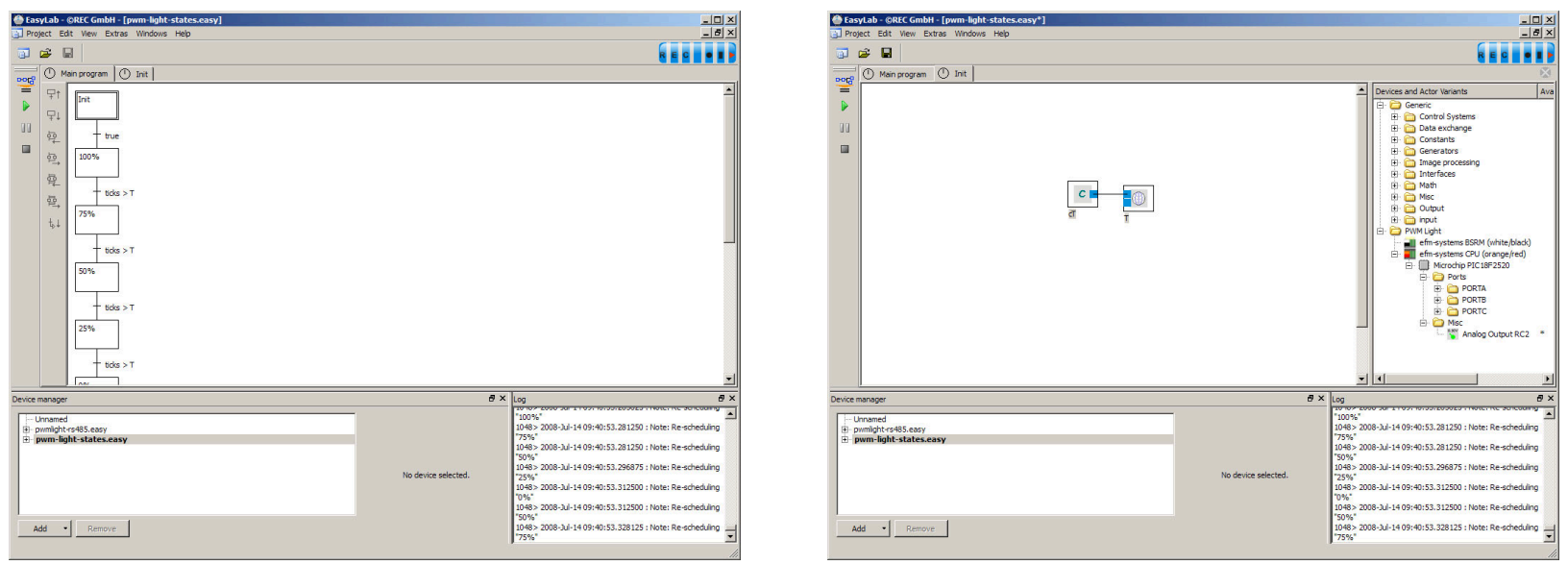

Figure 4. A sample application implemented in EasyLab with a SFC depicted on the left and a SDF shown on the right.

the serial port until the desired image brightness is achieved or the minimum or maximum possible brightness is reached.

The light's controller-board was designed to have a serial interface to receive brightness commands as plaintext values in a range from 0 to 255 , terminated by a linefeed. According to the read value, the microcontroller adjusts the pulse width modulated power signal for the ring-light which is mounted around the camera.

Figure 5 shows the controller-board on the left and the test setup for result verification on the right. The controller board basically consists of a Match-X stack with two modules, a power supply connector and connectors for a serial RS485 cable and the ring-light. The Match-X module which is marked black and white contains the voltage regulator for the CPU module and the RS485 serial interface, while the module which is color-coded in red and orange contains the CPU. The generated pulse width modulated signal is picked up through a wire directly from the databus and amplified by a MOSFET to match the ring-light's power specification. Figure 6 shows the light source with three different brightnesses.
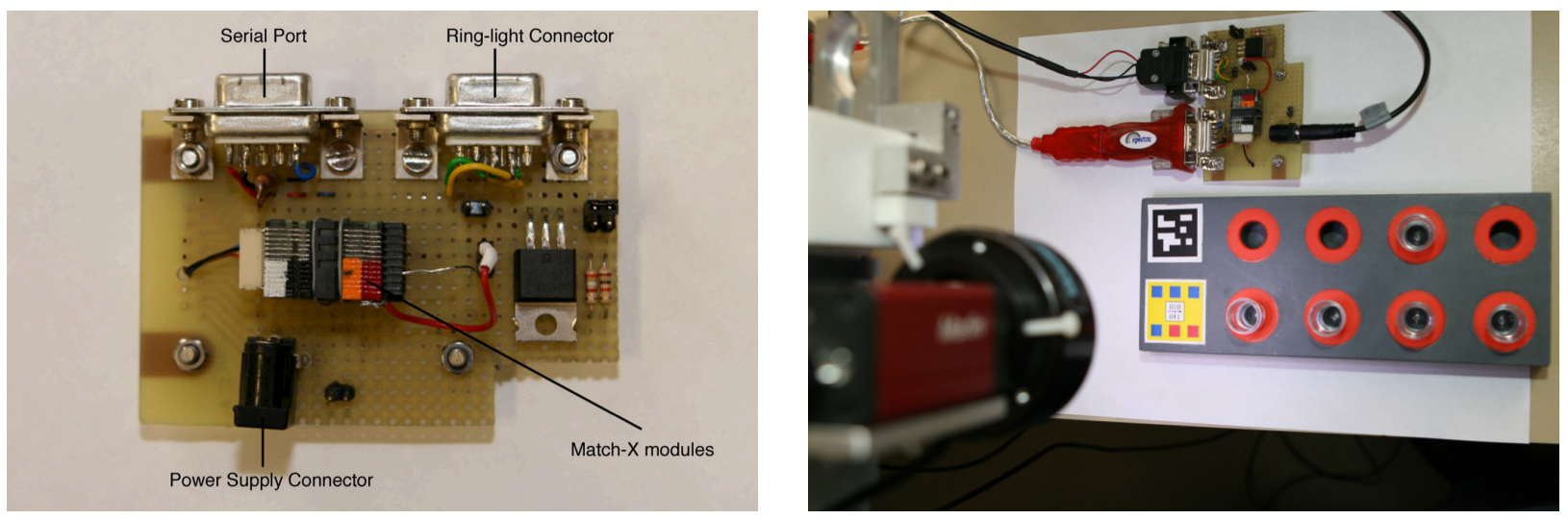

Figure 5. The controller board with the serial and the ring-light connector next to the Match-X stack consisting of two modules in this case. The right picture shows the mounted test setup for verifying the results.

\section{RESULTS}

To verify that the initial goal of improving illumination conditions for the image processing of the mobile robot was in fact achieved, we set up two series of experiments. The robot was placed in front of a lab workbench and 

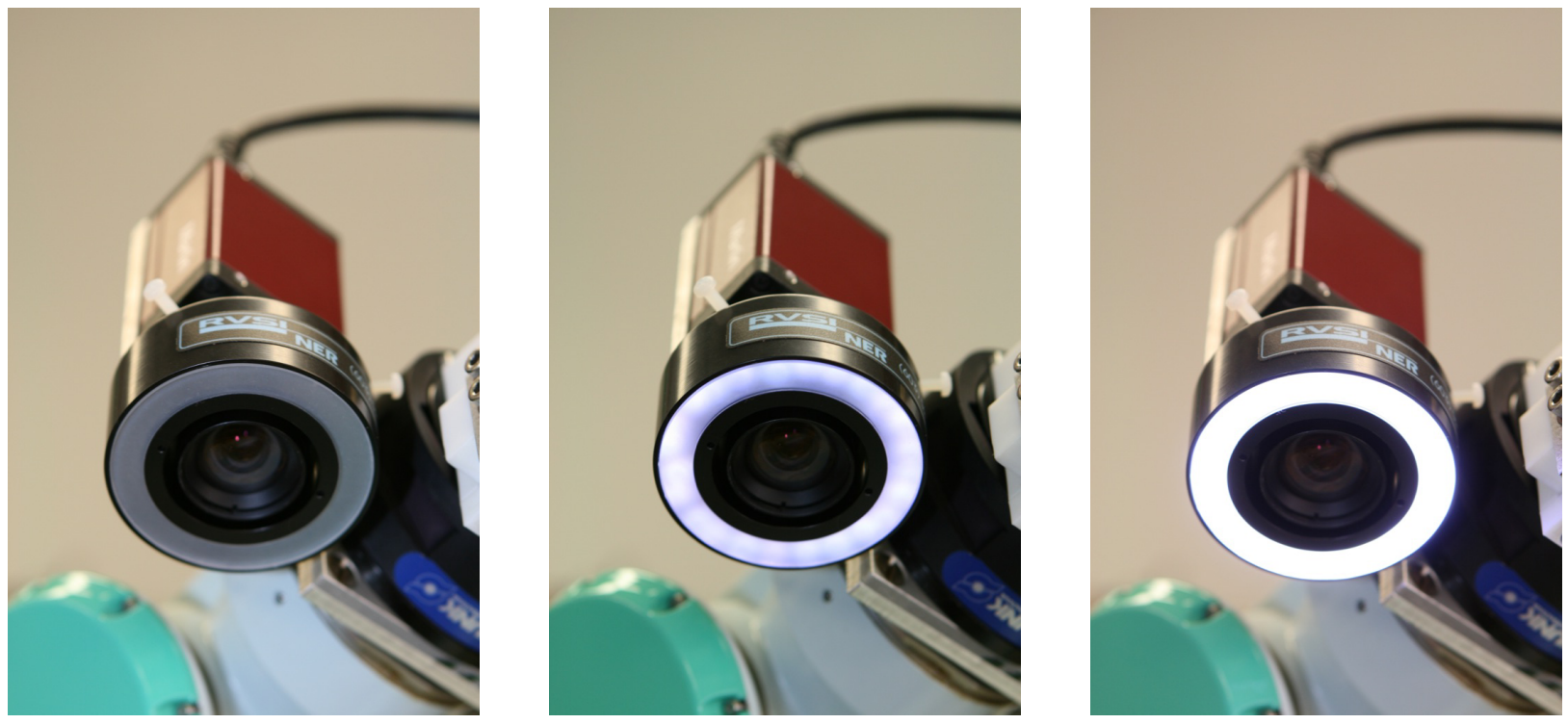

Figure 6. The light-source attached to the robot's tool in off-state, $30 \%$ and $100 \%$ brightness.
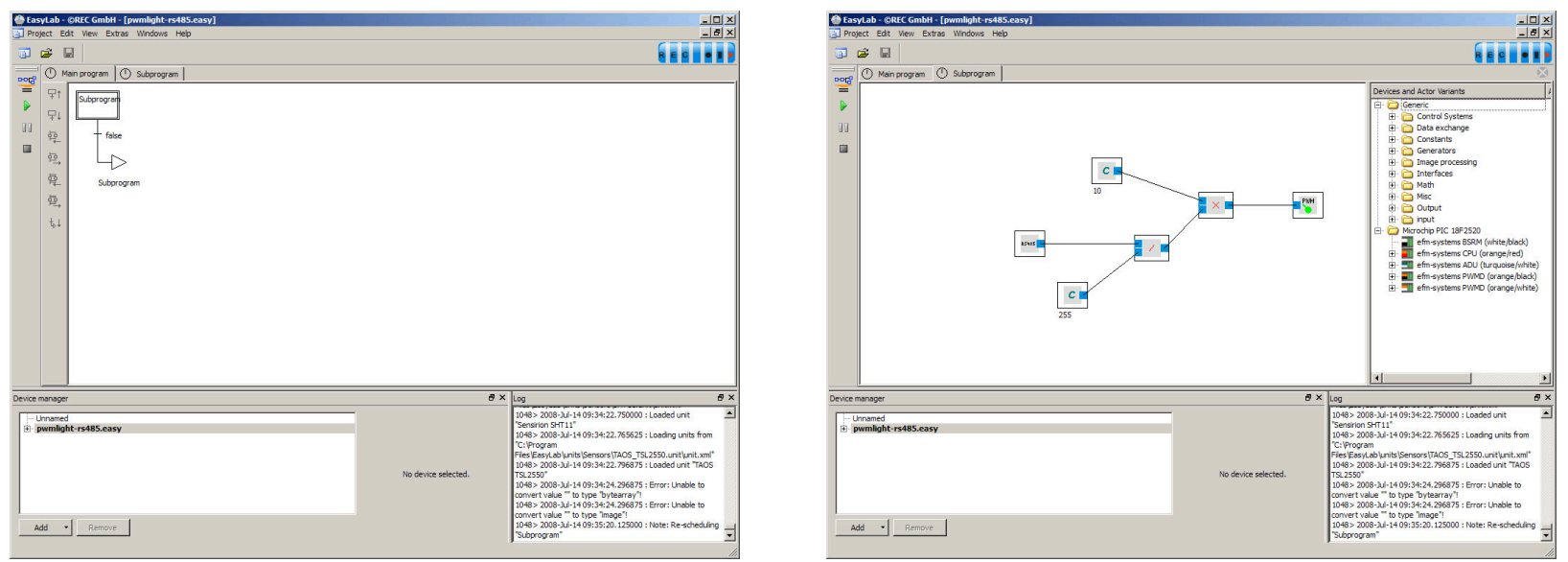

Figure 7. The software implementation of the adaptive light-source with EasyLab. The main program - on the left consists of a simple subroutine - shown on the right - with the necessary functional blocks, which can be easily arranged and connected by the user. The program reads a value in the range of 0 to 255 from the serial port, this value is converted into a floating point number through the division by 255 and is scaled up afterwards to a range of 0 to 10 which determines the frequency for the pulse width modulated power signal of the LED array. 
the arm was positioned above two different racks with vials. The racks have colored patches for calibrating the arm's position by calculating the centers of gravity of the color segments.

Figures 8 and 9 depict the experiments and the results. The first row of each Figure shows pictures taken at regular lab illumination with the ceiling lights switched on during daytime, while the second row in each figure shows pictures, which were taken, when the ceiling lights were switched off and the scene was illuminated by daylight only which entered through the lab windows. The third row of pictures were taken in darkness with almost no ambient illumination. The three columns in each figure distinguish between different brightness levels of the LED array, which were set to off, $30 \%$ and $100 \%$ brightness.

Every item in the figure consists of three pictures: the source image, which was taken with the robot's camera, the processed, color segmented picture below and the brightness histogram with a linear $\mathrm{x}$-axis and a logarithmic y-axis, representing dark values on the left and bright values on the right.

Taking a closer look at the results, and comparing the $0 \%$ column of the figures with the $33 \%$ and $100 \%$ ring-light brightness columns, it is apparent that the color segmentation works significantly better in the more challenging scenarios, where the ceiling lights in the lab are off. These were just three fixed illumination values for better comparability, however the robot's host application is actually implemented in an adaptive way, gradually changing the intensity of the light-source with respect to the mean of the brightness histogram to get optimal results. Furthermore it is possible to adjust the light appropriately, if highlights on shiny surfaces are detected.

\section{FUTURE WORK}

While we demonstrated fully automated, robust sample management in a pilot plant carried out by the mobile robot previously, further ideas came up. An autonomous robot - when not busy with sample management can also carry out lab walkthroughs periodically by observing pumps, pipes, tubes and motor drives utilized for continuous perfusion processes and trigger an alarm if devices fail to operate.

During power outages the battery driven robot equipped with its adaptive light-source can also be remote controlled to provide visual feedback in hazardous areas without sending people. At night, lab personnel can send the robot to certain stations to determine that experiments are carried out properly and not interrupted by defective devices.

The mentioned surveillance applications for this robot platform are actually evaluated intensively right now. Advanced image and video processing techniques will most likely be utilized to put some of the ideas into effect very soon. ${ }^{20}$ The developed computer-controllable light-source contributes significantly to the autonomy and versatility of the robot.

\section{CONCLUSIONS}

Autonomous vehicles became popular during the DARPA racing challenges, however mobile platforms equipped with a manipulator for increased flexibility also have been used successfully in biotech laboratories for sample management as shown on the well-known ESACT meetings. Navigation and object recognition is carried out by the utilization of a mounted machine vision camera. The challenge however is the different illumination conditions in a large life science laboratory. Thus the development of an adaptive light source for the visual servoed mobile manipulator was indispensable. NERLITE/Siemens build LED based ring illuminators suitable for many purposes, yet, in our case, the brightness needs to be computer controllable depending on the currently observed object and the ambient light to prohibit highlights on shiny surfaces and to allow pattern matching and vial inspection even in dark areas of the lab. We are aware that computer interfaceable LED controllers are available off the shelf, but we want to point out, that developing custom mechatronic applications utilizing the hardware toolbox EasyKit based on Match-X in conjunction with our software toolbox EasyLab can reduce development resource requirements dramatically in terms of money, man-power and time. In the presented application one single business day was sufficient to implement the necessary LED controller, which receives brightness commands via the serial port of a computer, depending on the visual feedback received from the connected camera. In our opinion many developers of mechatronic applications - not only for illumination purposes - would benefit of including EasyKit and EasyLab into their toolchain for rapid prototyping. 
$0 \%$
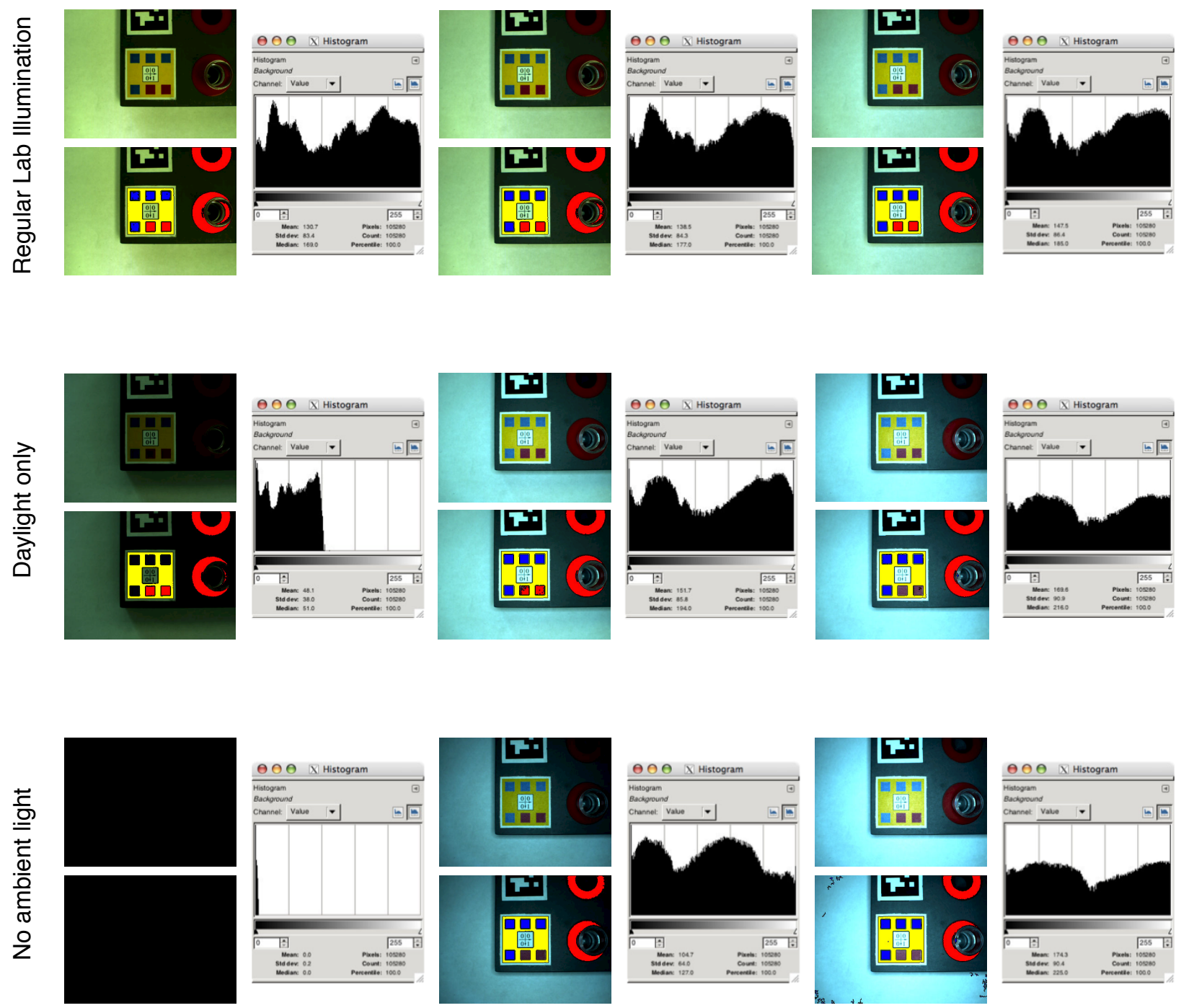

Figure 8. Color segmentation at the Cedex rack. While the color segmentation shows very good results in all cases when the ceiling lights are switched on in the lab, it becomes much harder to examine the colors in a lab when the ceiling lights are off, be it day or night. The LED array significantly improves color detection in the latter cases. 
$0 \%$
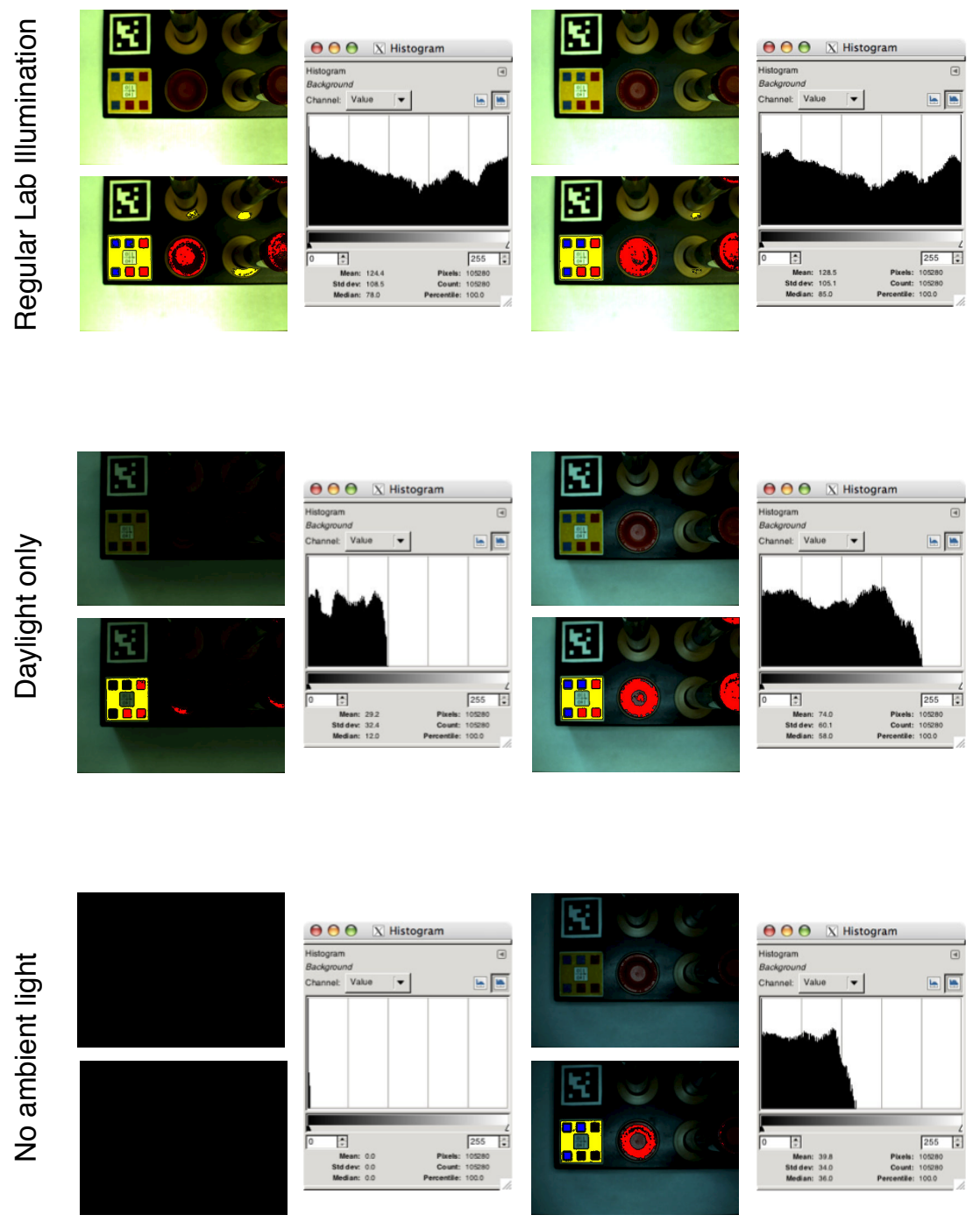

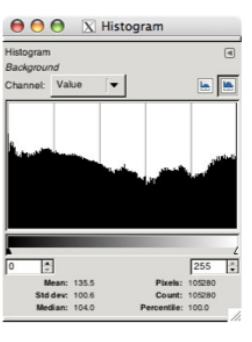

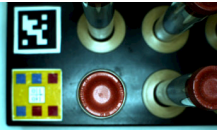
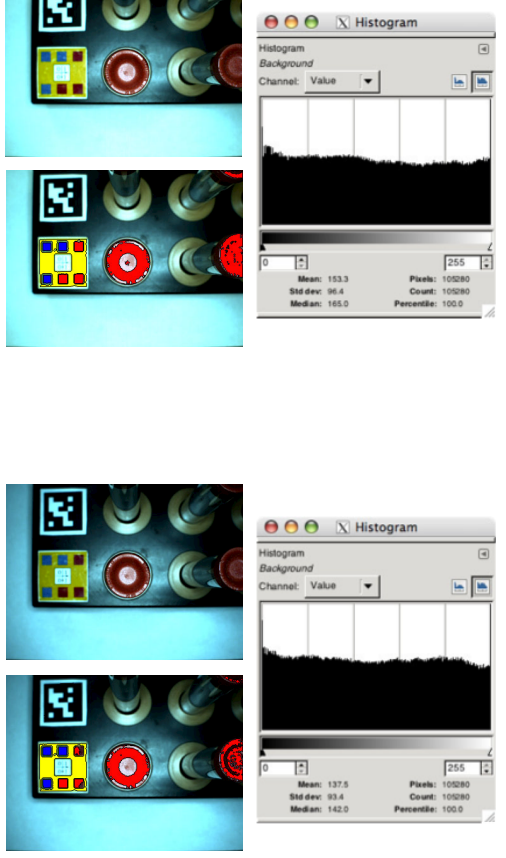

Figure 9. Color segmentation at the Vacutainer rack. The color segmentation shows constantly very good results if the ceiling lights in the lab are on. In the darker scenarios color segmentation works significantly better, with the additional illumination by the LED array. 


\section{ACKNOWLEDGMENTS}

This work is funded by the German Ministry of Education and Research.

\section{REFERENCES}

[1] Wojtczyk, M., Heidemann, R., Joeris, K., Zhang, C., Burnett, M., Knoll, A., and Konstantinov, K., "The use of a mobile robot for complete sample management in a cell culture pilot plant," in [Cell Technology for Cell Products, Proceedings of the 19th ESACT Meeting, Harrogate, UK, June 5-8, 2005], Smith, R., ed., 543-547, Springer Netherlands (2007).

[2] Siemens AG, NERLITE Machine Vision Illumination, Machine Vision Lighting and Machine Vision Imaging Products from Siemens. http://www.nerlite.com.

[3] Siemens AG, NERLITE R-60-1"V2" Series Ring. http://www.nerlite.com/PDFs/r-60-1-v2-series. pdf.

[4] Siemens AG, NERLITE R-70-2"V2" Series Ring. http://www.nerlite.com/PDFs/r-70-2-v2-series. pdf.

[5] Gardasoft Vision, Lighting Controllers for Machine Vision - Gardasoft. http://www.gardasoft.com/.

[6] Gardasoft Vision, PP610 - LED Lighting Controller with RS232 Control. http://www.gardasoft.com/ pdf/610Bro.pdf.

[7] Technische Universität München, EasyKit: Innovative Entwicklungs- und Didaktikwerkzeuge für mechatronische Systeme. http://easykit.informatik.tu-muenchen.de/.

[8] Lütkemeyer, D., Poggendorf, I., Scherer, T., Zhang, J., Knoll, A., and Lehmann, J., "First steps in robot automation of sampling and sample management during cultivation of mammalian cells in pilot scale," Biotechnology Progress 16(5), 822-828 (2000).

[9] Knoll, A., Scherer, T., Poggendorf, I., Lütkemeyer, D., and Lehmann, J., "Flexible Automation of Cell Culture and Tissue Engineering Tasks," Biotechnology Progress 20(6), 1825-1835 (2004).

[10] Poggendorf, I., Einsatz eines Serviceroboters zur Automatisierung der Probenentnahme und des Probenmangements während Kultivierungen tierischer Zellen in einer Technikumsumgebung, dissertation, Universität Bielefeld (June 2004).

[11] Scherer, T., A Mobile Service Robot for Automisation of Sample Taking and Sample Management in a Biotechnological Pilot Laboratory, dissertation, University of Bielefeld (Oct. 2004).

[12] Microchip Technology Inc., PIC18F2520. http://www.microchip.com.

[13] Verband Deutscher Maschinen- und Anlagenbau e.V. - Arbeitsgemeinschaft Match-X, VDMA - Einheitsblatt 66305: Bausteine und Schnittstellen der Mikrotechnik (July 2005). http://www.match-x.org/.

[14] VDMA, VDMA Homepage. http://www.vdma.org/.

[15] International Electrotechnical Commission, Norm EN 61131 (2003).

[16] Lee, E. A. and Messerschmitt, D. G., "Static scheduling of synchronous data flow programs for digital signal processing," IEEE Trans. Comput. 36(1), 24-35 (1987).

[17] Bhattacharyya, S. S., Lee, E. A., and Murthy, P. K., [Software Synthesis from Dataflow Graphs], Kluwer Academic Publishers, Norwell, MA, USA (1996).

[18] Bhattacharyya, S. S., Buck, J. T., Ha, S., and Lee, E. A., "Generating compact code from dataflow specifications of multirate signal processing algorithms," 452-464 (2002).

[19] Oh, H., Dutt, N., and Ha, S., "Memory optimal single appearance schedule with dynamic loop count for synchronous dataflow graphs," in [ASP-DAC '06: Proceedings of the 2006 conference on Asia South Pacific design automation], 497-502, IEEE Press, Piscataway, NJ, USA (2006).

[20] Panin, G., Lenz, C., Wojtczyk, M., Nair, S., Roth, E., Friedlhuber, T., and Knoll, A., "A unifying software architecture for model-based visual tracking," in [Image Processing: Machine Vision Applications. Edited by Niel, Kurt S.; Fofi, David. Proceedings of the SPIE, Volume 6813, pp. 681303-681303-14 (2008).], Presented at the Society of Photo-Optical Instrumentation Engineers (SPIE) Conference 6813 (Mar. 2008). 